\title{
A Produção Intelectual em Teoria Institucional: Análise nos Principais Periódicos Brasileiros a partir do Método Bibliométrico
}

\author{
Renato Fabiano Cintra \\ Universidade Nove de Julho, Uninove, Brasil \\ renatocintra@hotmail.com \\ Saulo Fabiano Amâncio-Vieira \\ Universidade Estadual de Londrina, UEL, Brasil \\ saulo@uel.br \\ Luciano Munck \\ Universidade Estadual de Londrina, UEL, Brasil \\ munck@uel.br
}

\section{RESUMO}

É difícil questionar a importância de estudos que debatem a teoria das organizações. Neste artigo, identificam-se o perfil dos autores que publicam sobre a teoria institucional no cenário nacional, bem como as obras relevantes e citadas, a partir da bibliometria. Trata-se de uma pesquisa exploratória de caráter quantitativo descritivo. Como resultados da pesquisa têm-se: 15 autores correspondem a 43,2\% da produção total e o restante, 56,8\% dos autores, publicou apenas uma vez. O perfil que produz conhecimento é composto pelas seguintes características: formação em administração ou engenharia; linha de pesquisa em estratégia organizacional ou estudos organizacionais ou administração pública; doutorado ou mestrado; gênero masculino; última titulação obtida entre 2003-2010; vínculo com a FGV/SP, PUC/PR, USP/SP ou UP/PR; e celetista ou servidor público. O estudo permite destacar as obras relevantes e citadas no campo e que a Revista de Administração Contemporânea (RAC) compõe o core no cenário nacional, pois tem $26,9 \%$ da publicação, seguida pela Brazilian Administration Review (BAR) com 19,4\%.

PALAVRAS-CHAVE: Teoria institucional. Neoinstitucional. Bibliometria. 
The Intellectual Production in Institutional Theory: Analysis of the

Main Brazilians Journals from Bibliometric Method

\section{ABSTRACT}

There is no disputing the importance of studies that discuss organizational theory. The article identifies the profile of authors who publish about institutional theory on the national scene, as well as relevant and cited works from the bibliometric. It is an exploratory descriptive quantitative character. How search results are up: 15 authors correspond to $43.2 \%$ of the total production and the rest, $56.8 \%$ of the authors only published once. The profile that produces knowledge consists of the following characteristics: training in management or engineering; line of research in organizational strategy or organizational or public administration studies; doctorate or master's degree; male gender; last titration obtained between 20032010; link to the FGV/SP, PUC/PR, USP/SP or UP/PR; and CLT or public servant. The study allows us to highlight the relevant works and cited in the field and that the RAC makes up the core on the national scene, it has $26.9 \%$ of the publication, followed by BAR with $19.4 \%$.

KEY-WORDS: Institutional theory. Neo-institutional. Bibliometric. 


\section{INTRODUÇÃO}

A teoria das organizações (TO) tem sido influenciada por mudanças sociais, políticas, econômicas e tecnológicas na sociedade como um todo (Rossetto \& Cunha, 1999). Em síntese, as teorias organizacionais propõem explicar (ou antever) fatos e acontecimentos, permitindo entender as práticas organizacionais (Mota, Beck, Pereira, Lima \& Vale, 2010). No cenário brasileiro, desde o trabalho seminal de Machado-da-Silva, Cunha e Amboni (1990), diversas análises sobre as publicações científicas foram realizadas (Rossoni, 2006) e continuam atraindo interesse dos pesquisadores em diversos segmentos, como é o caso da teoria do stakeholder (Cintra, Amâncio-Vieira, Suzuki \& Costa, 2014), dos sistemas de informação na administração pública (Oliveira et al., 2015), da tomada de decisão estratégica (Ribeiro \& Serra, 2014), da prospecção tecnológica (Yoshida, 2010); das ferramentas de e-serviços (Zittei \& Fernandes, 2015), da qualidade da produção científica brasileira (Cunha, Defina \& Passador, 2014), entre outros.

Logo, é difícil questionar a importância de estudos que debatem TO e suas ramificações, especialmente aquelas que buscam identificar relevância, pesquisadores, centros de pesquisa, campo e direções futuras (gaps). Destaca-se que em tempos recentes os estudos organizacionais experimentam vigoroso impulso no cenário brasileiro, tendo acumulado razoável produção científica (Caldas \& Bertero, 2007). Entretanto, após revisão dos trabalhos que empregavam teorias associadas aos estudos organizacionais, Mota et al. (2010) concluem, e alertam, que não há nada de novo, mas, sim, a replicação de teorias consolidadas internacionalmente. Desse modo, caminhos novos precisam ser percorridos.

Nesse contexto, destaca-se a teoria institucional (TI) no campo da TO, tendo em vista os fenômenos investigados. Ao considerar a publicação do clássico artigo de Meyer e Rowan (1977), "Institutionalized organizations: formal structure as myth and ceremony", como marco da perspectiva institucional, percebe-se incidência maior nas investigações dos fenômenos defendidos pela bandeira da TI (Quadro 1). 


\begin{tabular}{|l|l|}
\hline \multicolumn{1}{|c|}{ Fenômeno investigado } & \multicolumn{1}{c|}{ Principais autores } \\
\hline $\begin{array}{l}\text { 1. A expansão de políticas de pessoal } \\
\text { específicas }\end{array}$ & $\begin{array}{l}\text { Tolbert e Zucker (1983); Baron, Dobbin } \\
\text { e Jennings (1986); Edelman (1992) }\end{array}$ \\
\hline $\begin{array}{l}\text { 2. A redefinição da missão organizacional e de } \\
\text { suas estruturas }\end{array}$ & DiMaggio (1991); Fligstein (1985) \\
\hline $\begin{array}{l}\text { [até] 3. A formação de políticas nacionais e } \\
\text { internacionais por organizações governamentais }\end{array}$ & Strang (1990); Zhou (1993) \\
\hline
\end{tabular}

\section{Quadro 1: Fenômenos investigados e principais autores da teoria institucional}

Fonte: Elaborado pelos autores a partir da revisão teórica de Tolbert \& Zucker (1999)

Em síntese, a teoria institucional defende que, no início do ciclo de vida de um campo organizacional, as empresas têm variadas formas e estruturas. À medida que o campo vai se estruturando, a tendência gerada é por uma forte homogeneização (Diniz, Junquilho \& Carrieri, 2003). As organizações agem não somente por uma força burocrática à procura de eficiência, mas também por práticas culturais (Vieira \& Gomes, 2014) e é por meio institucional que os indivíduos sobressocializados aceitam e seguem normas sociais unicamente baseados em interesses (Berger \& Luckmann, 2011). As organizações concorrem pela legitimidade e aceitação ambiental, que se torna conceito central no institucionalismo. Atribui-se à legitimidade (adequação e aceitabilidade dos valores sociais) papel importante e equivalente à eficiência técnica (DiMaggio \& Powell, 1983). Desse modo, o foco da abordagem institucional tem sido, tradicionalmente, na forma pela qual os atores seguem persistentes scripts institucionais; questionamentos sobre como tais scripts são produzidos, mantidos e modificados têm sido amplamente negligenciados (Tolbert \& Zucker, 1999).

Ao ressaltar o papel das influências normativas nos processos de tomada de decisão organizacional, a teoria institucional oferece uma extensão importante e distintiva ao repertório de perspectivas e abordagens para explicar a estrutura organizacional (Tolbert \& Zucker, 1999). Logo, a teoria institucional não é um conjunto de proposições que vise especificamente à análise organizacional; pelo menos, a ela não se restringe (Machado-da-Silva \& Gonçalves, 1999). Três grandes pilares predominam entre os institucionalistas: o regulativo, o normativo e o cognitivo (Scott, 1995). Os pilares não tratam de posturas mutuamente exclusivas, mas de 
alternativas analíticas para compreensão de aspectos distintos do mesmo fenômeno (Machado-da-Silva \& Gonçalves, 1999).

Portanto, para que ocorra avanço nos campos de conhecimento, é necessário trabalho de investigação dos pesquisadores, alavancado por pesquisas científicas publicadas em periódicos, congressos e outros meios da academia. Um componente importante à elaboração deste estudo foi à análise bibliométrica, que se desenvolveu inicialmente a partir da elaboração de leis empíricas sobre o comportamento da literatura (Araújo, 2006). Assim, neste artigo objetiva-se: (i) identificar os artigos científicos que tratam da teoria institucional, publicados em periódicos nacionais com estrato Capes $A 1$ até B2, com vistas a traçar o perfil das autorias e coautorias por meio do levantamento e análise de seus Lattes (Plataforma Lattes do CNPq - MCT); e (ii) apresentar classificação e análise das obras citadas nas referências bibliográficas e no decorrer dos artigos, utilizando os recursos bibliométricos.

A justificativa de proceder à bibliometria de um campo científico centra-se na identificação das obras consideradas relevantes à discussão da teoria, bem como na facilitação da busca dos textos centrais, com vistas ao pesquisador iniciante ter a possibilidade de um "catálogo", para alcançar e/ou conhecer o estado da arte, neste caso, a teoria institucional no cenário nacional. Concomitantemente, é possível identificar colégios invisíveis, bem como a elite dos pesquisadores da teoria institucional no espaço geográfico estudado. Logo, deixando de lado os julgamentos de valor, parece clara a importância de dispor de uma distribuição que informe sobre o número de autores, trabalhos, países ou revistas que existem em cada categoria de produtividade, utilizada ou o que mais se deseja saber (Araújo, 2006).

O artigo inicia-se com uma introdução e objetivos, os quais já foram apresentados. Na segunda seção, explicita-se o suporte teórico com a discussão de bibliometria, leis clássicas e desdobramentos das aplicações em tempos recentes. $\mathrm{Na}$ sequência, apresentam-se os procedimentos metodológicos. Na seção quatro, discutem-se os resultados divididos em partes, pois delineiam-se o perfil e as características dos autores (titulação, gênero, instituição e vínculo); enquanto na outra seção abordam-se as citações e os textos mais relevantes a partir da análise bibliométrica. Por fim, 
são apresentadas as considerações, limitações e sugestões para pesquisas futuras.

\section{A BIBLIOMETRIA: CIÊNCIA DA INFORMAÇÃO}

A bibliometria é um conjunto de leis e princípios empíricos que contribuem para estabelecer os fundamentos teóricos da Ciência da Informação (Guedes \& Borschiver, 2005). Pao (1989, citado por Guedes \& Borschiver, 2005) acrescenta que publicações, autores, palavras-chave, usuários, citações e periódicos são alguns dos parâmetros observáveis em estudos bibliométricos, os quais tentam mensurar, descrever e prognosticar o processo de comunicação escrita. Assim, a bibliometria é uma ferramenta estatística usada para avaliação da produção científica e tecnológica, que permite mapear e gerar diferentes indicadores para tratamento e gestão da informação e do conhecimento (Guedes \& Boschiver, 2005). Existem, portanto, diversas leis e princípios bibliométricos que orientam os procedimentos de coleta e análise. De acordo com Araújo (2006) e Guedes e Borschiver (2005), as três leis clássicas e mais utilizadas são:

i. lei de Bradford: quantifica a produtividade de periódicos, descrevendo a distribuição da literatura periódica numa área específica (foco de estudo nos periódicos) e sua principal aplicação é estimar o grau de relevância de periódicos, em dada área do conhecimento;

ii. lei de Lotka: quantifica a produtividade científica de autores, descreve a produtividade dos autores (foco de estudo nos autores) e sua principal aplicação é estimar o grau de relevância de autores, em dada área do conhecimento;

iii. leis de Zipf: quantifica a frequência das palavras, descreve a frequência no uso de palavras num determinado texto (foco de estudo nas palavras) e sua principal aplicação é na indexação automática de artigos científicos e tecnológicos.

Dentre a distribuição dessas leis e princípios bibliométricos existe uma máxima a ser seguida e/ou respeitada: poucos com muito e muito com poucos (Guedes \& Borschiver, 2005). Para Merton (1968, citado por Guedes 
\& Borschiver, 2005), essa máxima é conhecida como o Efeito Mateus na Ciência, que diz: aos que mais têm, será dado em abundância; e, aos que menos têm, até o que têm lhes será tirado. Na sequência serão apresentadas as três leis clássicas, outros desdobramentos e aplicações da bibliometria em tempos recentes.

\subsection{A LEI DE LOTKA}

A Lei de Lotka é um princípio sobre a produtividade dos autores de determinado campo científico (Moretti \& Campanario, 2009). Lotka descobriu que uma larga proporção da literatura científica é produzida por um pequeno número de autores, e um grande número de pequenos produtores se iguala, em produção, ao reduzido número de grandes produtores (Araújo, 2006). Esta lei considera que alguns pesquisadores, supostamente de maior prestígio em determinada área do conhecimento, produzem muito e muitos pesquisadores, supostamente de menor prestígio, produzem pouco (Guedes \& Borschiver, 2005). No mesmo sentido, Moretti e Campanario (2009) afirmam que esta lei parte da premissa de que poucos autores publicam muito e são mais citados, enquanto muitos autores publicam menos e quase não são citados.

Para estabelecer a parte com que homens de diferentes qualidades contribuem ao progresso da ciência, Lotka (1926) estabeleceu os fundamentos da lei do quadrado inverso, afirmando que o número de autores que fazem $n$ contribuições em um determinado campo científico é aproximadamente $1 / \mathrm{n}^{2}$ daqueles que fazem uma só contribuição. A proporção daqueles que fazem uma única contribuição é de mais ou menos $60 \%$. (Alvarado, 2002, p. 14)

Assim, a relação entre o número de autores e o número de artigos publicados por esses, em qualquer área científica, segue a Lei do Inverso do Quadrado $1 / n^{2}$ (Guedes \& Borschiver, 2005). Segundo essa lei, se em dado período de tempo for analisado o número $n$ de artigos, o número de pesquisadores que escreveram dois artigos seria igual a $1 / 4$ do número de cientistas que escreveram um; logo, o número de pesquisadores que escreveram quatro artigos seria igual a $1 / 16$ do número de pesquisadores que escreveram apenas um, e assim por diante. 
Entre os aperfeiçoamentos realizados à lei de Lotka, destaca-se o trabalho de Price, que a partir de estudos realizados entre 1965 e 1971 concluiu que $1 / 3$ da literatura é produzida por menos de 1/10 dos autores mais produtivos, levando a uma média de 3,5 documentos por autor e $60 \%$ dos autores produzindo um único documento (Araújo, 2006). Vale ressaltar que Price (1965, citado por Guedes \& Borschiver, 2005) observou que, para as ciências em geral, o número de autores decresce mais rapidamente que o inverso do quadrado, mais aproximadamente à Lei do Inverso do Cubo $1 / n^{3}$. Portanto a aplicabilidade se verifica na avaliação da produtividade de pesquisadores, na identificação dos centros de pesquisas mais desenvolvidos, em dada área de assunto, e no reconhecimento da 'solidez' de uma área científica (Guedes \& Borschiver, 2005).

\subsection{A LEI DE BRADFORD}

Bradford realiza uma série de estudos que culminam, em 1934, com a formulação da lei da dispersão (Araújo, 2006). Verifica-se na Lei de Bradford, que permite estimar o grau de relevância de periódicos em dada área do conhecimento, que os periódicos que produzem o maior número de artigos sobre dado assunto formam um núcleo de periódicos, supostamente, de maior qualidade ou relevância para aquela área (Guedes \& Borschiver, 2005).

A Lei de Bradford sugere que, à medida que os primeiros artigos sobre um novo assunto são escritos, eles são submetidos a uma pequena seleção por periódicos apropriados e, se aceitos, esses periódicos atraem mais e mais artigos no decorrer do desenvolvimento da área de assunto (Guedes \& Borschiver, 2005). No mesmo sentido, Moran et al. (2010) relatam que, assim que os primeiros trabalhos sobre um novo tema são escritos e publicados em certos periódicos, esses veículos vão atrair mais artigos sobre o mesmo tema, o que ocasiona uma realimentação que acaba por construir uma imagem mais positiva de determinados periódicos para essa área de conhecimento. Pode-se inferir que criam uma "vocação institucional", tendo em vista a normalidade com que o assunto vem recorrer ao debate. 
Portanto, os periódicos devem ser listados com o número de artigos de cada um, em ordem decrescente, com soma parcial. O total de artigos deve ser somado e dividido por três; o grupo que tiver mais artigos, até o total de 1/3 dos artigos, é o core daquele assunto (Araújo, 2006). A Lei de Bradford é instrumento útil para o desenvolvimento de políticas de aquisição e de descarte de periódicos, em nível de gestão de sistemas de recuperação da informação, gestão da informação e do conhecimento científico e tecnológico (Guedes \& Borschiver, 2005).

\subsection{AS LEIS DE ZIPF}

As Leis de Zipf permitem estimar a frequência de ocorrência das palavras de determinado texto científico e tecnológico e a região de concentração de termos de indexação, ou palavras-chave. Um pequeno grupo de palavras ocorre muitas vezes e um grande número de palavras é de pequena frequência de ocorrência (Guedes \& Borschiver, 2005). Zipf descobriu que a palavra mais utilizada aparecia 2.653 vezes, a centésima palavra mais utilizada ocorria 256 vezes e a ducentésima palavra ocorria 133 vezes, em virtude disso, viu então que a posição de uma palavra multiplicada pela sua frequência era igual a uma constante de aproximadamente 26.500 (Araújo, 2006). Em um texto longo, existia relação entre a frequência com que dada palavra ocorria e sua posição na lista de palavras ordenadas segundo sua frequência de ocorrência e que o produto da ordem de série $(r)$ de uma palavra pela sua frequência de ocorrência $(f)$ era aproximadamente constante $(c)$, o que ficou conhecido como Primeira Lei de Zipf (Guedes \& Borschiver, 2005).

Tendo em vista que esta primeira lei é aplicável somente a palavras de alta frequência de ocorrência em um texto, Zipf propôs uma segunda lei, visando a palavras de baixa frequência de ocorrência, que foi revisada e modificada por Booth, em 1967 (Guedes \& Borschiver, 2005). Assim, a segunda lei de Zipf enuncia que, em determinado texto, várias palavras de baixa frequência de ocorrência (alta ordem de série) têm a mesma frequência. Booth, ao modificá-la, representou-a matematicamente da seguinte forma: 


$$
\frac{I_{1}}{I_{n}} \frac{n(n+1)}{2}
$$

em que $I_{1}$ é o número de palavras que têm frequência $1 ; I_{n}$ é o número de palavras que têm frequência n e 2, a constante válida para a língua.

Parte da literatura dedicada a esse tema tem se referido a essa segunda lei como a Lei de Zipf-Booth (Guedes \& Borschiver, 2005). Logo, as leis de Zipf vêm sendo aplicadas, inclusive, para identificar estilos distintos de autores, na redação de artigos científicos e tecnológicos (Guedes \& Borschiver, 2005).

\subsection{OUTROS DESDOBRAMENTOS E APLICAÇÕES DA BIBLIOMETRIA EM TEMPOS RECENTES}

A área mais importante da bibliometria é a análise de citações (Araújo, 2006). Citação é um conjunto de uma ou mais referências bibliográficas que, incluídas em uma publicação, evidenciam elos entre indivíduos, instituições e áreas de pesquisa, visto que mostram o relacionamento de uma publicação com outra (Foresti, 1989, citado por Araújo, 2006). A análise de citações permite não só identificar a frente de pesquisa de determinada área científica, por meio de um conjunto de autores que se citam na literatura recente, revelando um estreito padrão de relações múltiplas na literatura sobre o assunto; como também identificar, nesse pequeno grupo de artigos entrelaçados, o trabalho de algumas centenas de colaboradores que formam os colégios invisíveis (Guedes \& Borschiver, 2005).

A análise de citação pode ser definida como a parte da bibliometria que investiga as relações entre os documentos citantes e os documentos citados considerados como unidades de análise, no todo ou em suas diversas partes: autor, título, origem geográfica, ano, idioma de publicação, etc. (Foresti, 1989, citado por Araújo, 2006). A hipótese é de que, em determinada área científica, artigos de periódicos citados mais frequentemente são mais relevantes do que artigos menos citados (Guedes \& Borschiver, 2005). Logo, as citações visam, primordialmente, à acessibilidade ao material citado e indicam uso do citado pelo citante, 
podendo, ainda, contar artigos, periódicos, autores, departamentos acadêmicos, universidades, institutos de pesquisa, entre outros. Também um artigo de periódico muito citado representa a aceitação da comunidade que o cita (Guedes \& Borschiver, 2005). Dentro da bibliometria, particularmente a análise de citações permite a identificação e a descrição de uma série de padrões na produção do conhecimento científico.

Com os dados retirados das citações pode-se descobrir: autores mais citados, autores mais produtivos, elite de pesquisa, frente de pesquisa, fator de impacto dos autores, procedência geográfica e/ou institucional dos autores mais influentes em um determinado campo de pesquisa; tipo de documento mais utilizado, idade média da literatura utilizada, obsolescência da literatura, procedência geográfica e/ou institucional da bibliografia utilizada; periódicos mais citados, 'core' de periódicos que compõem um campo. (Araújo, 2006, p. 18-9)

A Lei do Elitismo enuncia que toda população de tamanho $N$ tem uma elite efetiva de tamanho $\sqrt{ } N^{\prime \prime}$ (Price, 1965, citado por Guedes \& Borschiver, 2005). Para Price (1965, citado por Araújo, 2006), o número de membros da elite corresponde à raiz quadrada do número total de autores, e a metade do total da produção é considerada o critério para se saber se a elite é produtiva ou não. E finalmente, embora não baseada na análise de citações, vale destacar a Lei dos 80/20, que consiste em um fenômeno, inicialmente observado no comércio e na indústria, segundo o qual em sistemas de informação $80 \%$ da demanda de informação se satisfaz com $20 \%$ do conjunto de fontes de informação (Guedes \& Borschiver, 2005).

\section{PROCEDIMENTOS METODOLÓGICOS}

Trata-se de uma pesquisa exploratória de caráter quantitativo descritivo e utiliza recursos bibliométricos. Para a coleta e a identificação dos dados necessários à pesquisa, foi realizado trabalho minucioso e rigoroso de verificação no banco de dados da Capes, onde foram selecionados periódicos de instituições nacionais classificadas no estrato Capes A1 a B2 (triênio 20102012). Para compor o Quadro 2, utilizaram-se apenas os periódicos que tiveram resultados. 


\begin{tabular}{|lccc|}
\hline \multicolumn{1}{|c|}{ Descrição do Periódico e Instituição } & $\begin{array}{c}\text { Institucional } \\
\text { ou } \\
\text { Institutional }\end{array}$ & Aborda & $\begin{array}{c}\text { Não } \\
\text { Aborda }\end{array}$ \\
\hline BAR - Brazilian Administration Review - ANPAD & 32 & 13 & 19 \\
\hline G\&P - Gestão \& Produção - UFSCAR & 20 & 1 & 19 \\
\hline RAP - Revista de Administração Pública - FGV & 45 & 2 & 43 \\
\hline RAC - Revista de Administração Contemporânea - ANPAD & 59 & 29 & 30 \\
\hline RAC-e - Revista de Administração Contemporânea Eletrônica - A & 7 & 0 & 7 \\
\hline RAE - Revista de Administração de Empresas - FGV & 24 & 8 & 16 \\
\hline RAE-e - Revista de Administração de Empresas Eletrônica - FGV & 13 & 2 & 11 \\
\hline RAM - Revista de Administração Mackenzie - UPM & 22 & 4 & 18 \\
\hline O\&S - Organizações \& Sociedade - UFBA & 33 & 6 & 27 \\
\hline RAUSP - Revista de Administração - USP & 39 & 2 & 37 \\
\hline REAd - Revista Eletrônica de Administração - UFRGS & 31 & 11 & 20 \\
\hline Total Geral ..... & $\mathbf{3 2 5}$ & $\mathbf{7 8}$ & $\mathbf{2 4 7}$ \\
\hline Percentual ..... & $\mathbf{1 0 0 \%}$ & $\mathbf{2 4 \%}$ & $\mathbf{7 6 \%}$ \\
\hline
\end{tabular}

\section{Quadro 2: Periódicos nacionais selecionados para compor a amostra do trabalho}

Fonte: Elaborado pelos autores, com base na coleta dos dados

A justificativa para selecionar apenas periódicos nacionais está no fato de que, no presente artigo, busca-se identificar a produção intelectual brasileira. Ainda, o corte busca abarcar os periódicos nacionais classificados no estrato Qualis Capes (triênio 2010-2012) com métricas melhores e, portanto, parte da premissa de que são publicações de maior relevância e impacto no cenário nacional.

Em seguida foi realizada busca em cada site dos periódicos, com a palavra institucional (em língua nacional e estrangeira - inglês) em qualquer campo do artigo (título, resumo e palavra-chave), perfazendo um total de 325 artigos que apresentavam a expressão-chave. A partir daí foi realizada a leitura de cada resumo e título dos tópicos nos referidos artigos, selecionando assim aquele que trouxesse o assunto pertinente ou alguma discussão da teoria institucional, o que reduziu a amostra para 78 artigos, conforme Quadro 2 (coluna Aborda).

Deste total de artigos, 11 foram republicados na Revista de Administração Contemporânea (RAC) em edições especiais, fazendo com que a amostra final ficasse em 67 artigos para serem analisados, conforme Quadro 3. Os artigos foram numerados de 1 a 67 , com seus respectivos títulos, revistas, ano de publicação e arquivados em meio digital (extensão *.pdf). Na sequência, os artigos foram classificados conforme os periódicos e 
os referidos anos em que foram publicados, demonstrando a linha do tempo da discussão da teoria institucional no cenário nacional, conforme Quadro 3.

\begin{tabular}{|c|c|c|c|c|c|c|c|c|c|c|c|c|c|c|}
\hline Revista e Instituição & 1999 & 2000 & 2001 & 2002 & 2003 & 2004 & 2005 & 2006 & 2007 & 2008 & 2009 & 2010 & 2011 & Total \\
\hline BAR - ANPAD & - & - & - & - & - & - & 2 & 2 & 5 & 1 & 1 & 2 & - & 13 \\
\hline G\&P - UFSCAR & - & - & - & 1 & - & - & - & - & - & - & - & - & - & 1 \\
\hline RAP - FGV & - & - & - & - & - & - & - & - & - & - & - & 1 & 1 & 2 \\
\hline RAC - ANPAD & - & - & 2 & 1 & - & 3 & - & 3 & 3 & - & - & 5 & 1 & 18 \\
\hline RAE - FGV & - & - & - & - & 1 & - & 2 & 1 & 1 & 2 & - & 2 & 1 & 10 \\
\hline RAM - UPM & - & - & - & - & - & - & 1 & 1 & - & - & - & - & 2 & 4 \\
\hline O\&S - UFBA & - & - & - & 1 & - & - & 1 & - & - & 1 & 1 & - & 2 & 6 \\
\hline RAUSP - USP & - & - & - & - & - & - & - & - & - & - & - & 1 & 1 & 2 \\
\hline REAd - UFRGS & 5 & - & 3 & - & - & - & 1 & - & - & - & 2 & - & - & 11 \\
\hline Sub-Total & 5 & 0 & 5 & 3 & 1 & 3 & 7 & 7 & 9 & 4 & 4 & 11 & 8 & 67 \\
\hline $\begin{array}{l}\text { RAC - ANPAD } \\
\text { (republicados) }\end{array}$ & - & - & - & - & - & - & 2 & 2 & 3 & - & - & 4 & - & 11 \\
\hline Total Geral & 5 & $\mathbf{0}$ & 5 & 3 & 1 & 3 & 9 & 9 & 12 & 4 & 4 & 15 & 8 & 78 \\
\hline
\end{tabular}

\section{Quadro 3: Publicações em teoria institucional nos periódicos nacionais por ano}

Fonte: Elaborado pelos autores, com base na coleta dos dados

Subsequentemente, foram identificados e relacionados todos os autores e coautores dos 67 artigos com o objetivo de traçar o perfil dos pesquisadores que publicaram artigos científicos com assuntos relacionados à teoria institucional. Para isso, os dados dos autores e coautores foram tratados mediante o levantamento das informações no Lattes, disponibilizado pelo Ministério da Ciência e Tecnologia (MCT) e denominado Plataforma de Currículo Lattes.

Os perfis foram relacionados em planilha eletrônica (Excel 2010), com as seguintes informações: nome do pesquisador, maior titulação concluída, ano de obtenção da maior titulação, linha de pesquisa (a primeira informada), instituição e vínculo. Esses dados foram trabalhados com o fim de mostrar indicadores agregados que demonstrassem o perfil dos pesquisadores, bem como instituições às quais estão vinculados e a concentração da produção científica. Para os pesquisadores que não possuíam Currículo Lattes disponível na plataforma analisada, foi estabelecida a titulação e a instituição que constava nas informações do autor no referido artigo (quando constava) e o restante das informações foi desconsiderado (ano de obtenção da maior titulação, linha de pesquisa e vínculo institucional).

E por último, organizou-se a construção de um banco de dados sobre as obras citadas pelos autores dos artigos, os quais foram colhidos um a um. 
O nome de cada uma das obras citadas (referências bibliográficas) foi relacionado em planilha eletrônica (Excel 2010) e, pelo seu sistema de filtro, procedeu-se ao somatório das citações apresentadas em cada artigo e total geral, bem como a quantidade que a obra foi relacionada no total dos artigos analisados. Importante assinalar que as obras que continham mesmo autor e anos diferentes foram agrupadas, pois tratam do mesmo assunto, atualizado e/ou revisado.

Pelo fato de existirem dois diferentes tipos de autores a serem considerados, adotaram-se a denominação autores para aqueles que produziram os artigos pesquisados nos periódicos nacionais e a denominação obras citadas ou citações aos trabalhos e seus autores citados nas referências. O primeiro grupo compõe o objeto de estudo do perfil dos autores, enquanto o segundo é objeto de estudo bibliométrico, como será apresentado no próximo tópico.

\section{RESULTADOS E DISCUSSÃO}

No intuito de atingir os objetivos do artigo, este item será subdividido em dois, a primeira parte será destinada a apresentar o perfil dos autores dos artigos analisados e a segunda ao mapeamento geral das obras citadas.

\subsection{PERFIS DOS AUTORES}

Esta seção está dividida em: (i) áreas de formação dos autores; (ii) linhas de pesquisa; (iii) gênero e maior titulação dos autores dos artigos analisados; (iv) instituição e vínculo; e, por fim, (v) autoria e coautoria.

\subsection{1 Áreas de formação dos autores}

O primeiro objetivo foi traçar o perfil dos autores por meio do Currículo Lattes. Como esperado, por se tratar de um assunto ligado ao contexto organizacional, a maioria dos autores possui formação dentro da grande área das ciências sociais aplicadas, compreendendo 77 autores, ou seja, 78,5\% do total de 98 autores (já excluídas as repetições); os restantes 
$21,5 \%$ estão divididos em: engenharia $(9,2 \%)$, ciências sociais $(2 \%)$, outros $(5,1 \%)$ e os que não possuíam Lattes ou informação $(6,1 \%)$. Na Tabela 1 , apresentam-se todas as áreas encontradas a partir dos dados analisados com as referidas quantidades.

\section{Tabela 1: Áreas de formação dos autores}

\begin{tabular}{ll|c|c|c}
\hline \multicolumn{1}{c|}{ Descrição } & Qtde. & \%Freq. & \%Acum. \\
\hline $\mathbf{1}$ Administração & & 71 & $72,4 \%$ & $72,4 \%$ \\
$\mathbf{2}$ Engenharia & & 9 & $9,2 \%$ & $81,6 \%$ \\
$\mathbf{3}$ Contabilidade & 5 & $5,1 \%$ & $86,7 \%$ \\
$\mathbf{4}$ Ciências Sociais & 2 & $2,0 \%$ & $88,8 \%$ \\
$\mathbf{5}$ Outros (Ciência da Informação; Ciência Política; Economia; Letras e Zootecnia) & 5 & $5,1 \%$ & $93,9 \%$ \\
$\mathbf{6}$ Sem informação & 6 & $6,1 \%$ & $100,0 \%$ \\
\hline \multicolumn{2}{c|}{ Total } & $\mathbf{9 8}$ & $\mathbf{1 0 0 \%}$ \\
\cline { 1 - 3 }
\end{tabular}

Fonte: Elaborada pelos autores, com base na coleta dos dados

Vale ressaltar ainda que, quando relacionados, os autores e coautores dos 67 artigos totalizam 146 autorias, das quais, 98 eram autores diferentes e o restante (48) foi repetido. Em outras palavras, os autores (ou coautores) trabalharam conjuntamente em outros artigos. Desse total de 146 autores e/ou coautores, apenas 15 que apareceram com mais de uma publicação e juntos correspondem a 43,2\% das publicações. A formação destes 15 autores é: administração (10), contabilidade (2), engenharia (2) e ciências sociais (1). Destaca-se que esse procedimento foi adotado tendo em vista que alguns autores informam que a contagem direta e a contagem ajustada não produzem diferenças essenciais e que os dois meios de contagem produzem a mesma coisa, por isso não seria necessário considerar a contagem ajustada e se deveria prestar maior atenção à contagem direta (Nath \& Jackson, 1991, citados por Alvarado, 2002).

\subsubsection{Linhas de pesquisas}

Em relação às linhas de pesquisas dos 98 autores, foi identificado que 69 destes possuíam linhas definidas e identificáveis (70\% dos autores). Destaca-se que sete autores não possuíam Lattes (enquadrados como sem informação). Foi adotado como critério o primeiro registro de linha de pesquisa que constava no currículo. Após a identificação das linhas de 
pesquisas, foi realizado o agrupamento em linhas de concentração mais genéricas para facilitar a identificação do perfil dos pesquisadores, conforme Tabela 2.

Tabela 2: Linhas de pesquisas dos autores

\begin{tabular}{|c|c|c|c|c|}
\hline \multicolumn{2}{|r|}{ Descrição } & \multirow{2}{*}{$\frac{\text { Qtde. }}{24}$} & \multirow{2}{*}{$\begin{array}{c}\text { \%Freq. } \\
24,5 \%\end{array}$} & \multirow{2}{*}{\begin{tabular}{|c|} 
\%Acum. \\
$24,5 \%$
\end{tabular}} \\
\hline 1 & Estratégia Organizacional & & & \\
\hline 2 & Estudos Organizacionais e Teoria Organizacional & 10 & $10,2 \%$ & $34,7 \%$ \\
\hline 3 & Administração Pública & 9 & $9,2 \%$ & $43,9 \%$ \\
\hline 4 & Administração Geral & 8 & $8,2 \%$ & $52,0 \%$ \\
\hline 5 & Gestão de Pessoas & 6 & $6,1 \%$ & $58,2 \%$ \\
\hline 6 & Marketing e Empreendedorismo & 5 & $5,1 \%$ & $63,3 \%$ \\
\hline 7 & Sistema de Informação e Tecnologia da Informação & 4 & $4,1 \%$ & $67,3 \%$ \\
\hline 8 & Planejamento, Controle e Produção & 4 & $4,1 \%$ & $71,4 \%$ \\
\hline 9 & Contabilidade & 3 & $3,1 \%$ & $74,5 \%$ \\
\hline 10 & Comunicação e Mudança Organizacional & 3 & $3,1 \%$ & $77,6 \%$ \\
\hline 11 & Outras Linhas & 15 & $15,3 \%$ & $92,9 \%$ \\
\hline 12 & Sem Informação & 7 & $7,1 \%$ & $100,0 \%$ \\
\hline \multicolumn{2}{|r|}{ Total } & 98 & $100 \%$ & \\
\hline
\end{tabular}

Fonte: Elaborada pelos autores, com base na coleta dos dados

Estratégia organizacional obteve a maior frequência com 24,5\%, seguida de estudos organizacionais com 10,2\% e administração pública com 9,2\%. Juntas perfazem $43,9 \%$ da frequência total, o que representa a maioria dos pesquisadores. Tais achados eram esperados, haja vista que a discussão da teoria institucional passa por questões ligadas à estratégia organizacional, às instituições (principalmente as públicas com a questão do isomorfismo e do aparato institucional) e ao próprio estudo organizacional que é discutido em TO.

Percebe-se também a pluralidade e a possibilidade de convergência e discussão da teoria institucional nos campos, pois foram encontradas linhas como: administração geral, gestão de pessoas, marketing e empreendedorismo, sistema de informação e tecnologia da informação, planejamento, controle e produção, contabilidade, comunicação e mudança organizacional. Vale destacar que a descrição "outras linhas" foi composta por várias linhas que apareceram apenas uma vez e foram agrupadas para demonstrar o quão diversificadas foram, perfazendo um total de 15 linhas 
distintas e representando $15,3 \%$ do total. Também, os resultados reafirmam que estudos em teorias organizacionais são frutos de autores que se identificam e têm interesses na melhoria da teoria das organizações, acumulando aproximadamente $52 \%$ de toda a produção.

\subsubsection{Gênero e titulação dos autores}

O perfil por gênero dos autores e coautores dos artigos dentro do universo pesquisado foi retirado de seus Lattes, os dados evidenciam o desequilíbrio entre homens e mulheres, pois os homens correspondem a $69 \%$ dos autores, enquanto as mulheres, a 31\%. Os homens são os que possuem a maior titulação com $12 \%$ de pós-doutorado e 36\% doutorado, enquanto as mulheres são representadas por $3 \%$ de pós-doutoras e $17 \%$ de doutoras (Tabela 3).

Tabela 3: Maior titulação e gênero dos autores

\begin{tabular}{ll|c|c|c|c|c|c|c}
\hline & Titulação & Masc. & \%Freq. & Fem. & \%Freq. & Total & \%Freq. & \%Acum. \\
\hline $\mathbf{1}$ & Doutorado & 35 & $36 \%$ & 17 & $17 \%$ & 52 & $53 \%$ & $53 \%$ \\
$\mathbf{2}$ & Mestrado & 10 & $10 \%$ & 10 & $10 \%$ & 20 & $20 \%$ & $73 \%$ \\
$\mathbf{3}$ & Pós-Doutorado & 12 & $12 \%$ & 3 & $3 \%$ & 15 & $15 \%$ & $89 \%$ \\
$\mathbf{4}$ & Livre-Docência & 5 & $5 \%$ & 0 & $0 \%$ & 5 & $5 \%$ & $94 \%$ \\
$\mathbf{5}$ & Graduação & 1 & $1 \%$ & 0 & $0 \%$ & 1 & $1 \%$ & $95 \%$ \\
$\mathbf{6}$ & Sem informação & 5 & $5 \%$ & 0 & $0 \%$ & 5 & $5 \%$ & $100 \%$ \\
\hline \multicolumn{2}{r|}{ Total } & $\mathbf{6 8}$ & $\mathbf{6 9 \%}$ & $\mathbf{3 0}$ & $\mathbf{3 1 \%}$ & $\mathbf{9 8}$ & $\mathbf{1 0 0} \%$ &
\end{tabular}

Fonte: Elaborada pelos autores, com base na coleta dos dados.

Nota-se que mais de $50 \%$ da produção é proveniente de pesquisas realizadas por doutores e, se somada aos pós-doutores, atinge o percentual de $68 \%$. Ressalta-se aqui a importância das pesquisas de cunho qualificado com o aumento dos programas de pós-graduação que formam doutores e pós-doutores no cenário nacional, além da necessidade de não só replicar teorias consagradas internacionalmente, mas em produzir TO brasileira.

Ao se analisarem a titulação dos autores e o ano de obtenção comparado com as publicações em periódicos nacionais (Tabela 4), pode-se inferir que, em regra, os artigos publicados são frutos dos programas de pósgraduação stricto sensu (doutorado e mestrado), tendo em vista que ao longo 
do período foram publicados 67 artigos, enquanto um total de 62 doutores e mestres foram titulados (1999-2011). Esse achado é corroborado por Rossoni (2006), que afirma que o processo de desenvolvimento de pesquisas e de treinamento de novos pesquisadores se dá por meio de programas de mestrado e doutorado, sendo influenciado principalmente pelas relações do professor com o aluno.

Tabela 4: Maior titulação dos autores e ano de obtenção

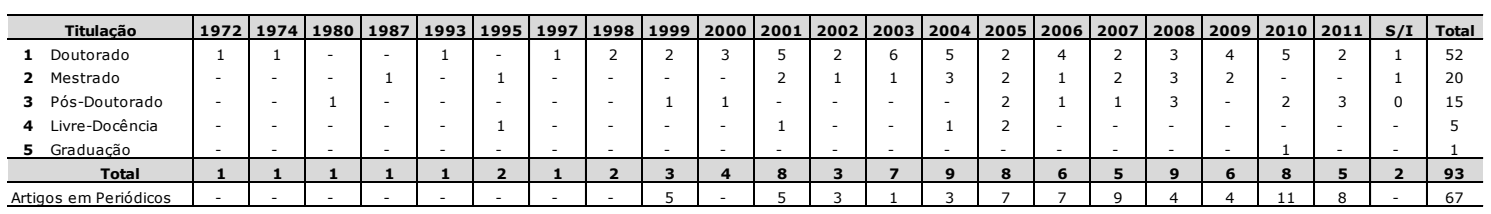

Fonte: Elaborado pelos autores, com base na coleta dos dados

A maior incidência de publicações foi nos períodos de 2001, 20052007 e 2010-2011, totalizando 70\%, similar às obtenções dos títulos que, nos períodos de 2001 e 2003-2011, corresponderam a 76\% do quantitativo geral. O que perfaz uma relação próxima de 1,19 título de mestrado ou doutorado para cada artigo publicado em periódicos nacionais classificados pela Capes no mesmo período. Com base na Tabela 4, os três melhores anos de publicação da teoria institucional foram: 2010 com 11 artigos, 2007 com nove e 2011 com oito.

\subsubsection{Instituição e vínculo}

No que tange às instituições que contribuíram com a discussão em nível nacional, podem-se destacar 16 , que juntas somam $66,3 \%$ dos autores, conforme Tabela 5. 
Tabela 5: Instituição informada pelo autor

\begin{tabular}{rl|c|c|c}
\hline \multicolumn{1}{c|}{ Instituição } & Qtde. & \%Freq. & \%Acum. \\
\hline $\mathbf{1}$ & Fundação Getúlio Vargas - FGV/SP & 7 & $7,1 \%$ & $7,1 \%$ \\
$\mathbf{2}$ & Pontifícia Universidade Católica do Paraná - PUC/PR & 7 & $7,1 \%$ & $14,3 \%$ \\
$\mathbf{3}$ & Universidade de São Paulo - USP & 7 & $7,1 \%$ & $21,4 \%$ \\
$\mathbf{4}$ & Universidade Positivo - UP/PR & 7 & $7,1 \%$ & $28,6 \%$ \\
$\mathbf{5}$ & Universidade Federal do Rio Grande do Sul - UFRGS & 6 & $6,1 \%$ & $34,7 \%$ \\
$\mathbf{6}$ & Fundação Getúlio Vargas - FGV/RJ & 4 & $4,1 \%$ & $38,8 \%$ \\
$\mathbf{7}$ & Universidade Federal de Pernambuco - UFPE & 4 & $4,1 \%$ & $42,9 \%$ \\
$\mathbf{8}$ & Universidade de Brasília - UnB & 3 & $3,1 \%$ & $45,9 \%$ \\
$\mathbf{9}$ & Universidade do Estado de Santa Catarina - UESC & 3 & $3,1 \%$ & $49,0 \%$ \\
$\mathbf{1 0}$ & Universidade Estadual de Maringá - UEM & 3 & $3,1 \%$ & $52,0 \%$ \\
$\mathbf{1 1}$ & Universidade Federal de Minas Gerais - UFMG & 3 & $3,1 \%$ & $55,1 \%$ \\
$\mathbf{1 2}$ & Universidade Federal do Rio de Janeiro - UFRJ & 3 & $3,1 \%$ & $58,2 \%$ \\
$\mathbf{1 3}$ & Pontifícia Universidade Católica do Rio de Janeiro - PUC/RJ & 2 & $2,0 \%$ & $60,2 \%$ \\
$\mathbf{1 4}$ & Universidade do Vale do Itajaí - UNIVALI/SC & 2 & $2,0 \%$ & $62,2 \%$ \\
$\mathbf{1 5}$ & Universidade Federal da Bahia - UFBA & 2 & $2,0 \%$ & $64,3 \%$ \\
$\mathbf{1 6}$ & Universidade Federal de Juiz de Fora - UFJF/MG & 2 & $2,0 \%$ & $66,3 \%$ \\
$\mathbf{1 7}$ & Outras Instituições & 32 & $32,7 \%$ & $99,0 \%$ \\
$\mathbf{1 8}$ Sem Informação & 1 & $1,0 \%$ & $100 \%$ \\
\hline & $\mathbf{9 8}$ & $\mathbf{1 0 0 \%}$ & \\
\cline { 1 - 2 } & Total &
\end{tabular}

Fonte: Elaborada pelos autores, com base na coleta dos dados

Ao analisar os autores que publicaram no mínimo duas vezes com temas ligados à teoria institucional, totalizando 15 autores, pode-se inferir que: três são da Universidade Positivo (UP/PR), um da Universidade Estadual de Maringá (UEM), um da Universidade Federal do Rio Grande do Sul (UFRGS), um da Pontifícia Universidade Católica do Paraná (PUC/PR), dois da Fundação Getúlio Vargas (FGV/RJ), um da Universidade Fumec (BH/MG); dois da Universidade de São Paulo (FEA/USP), dois da Fundação Getúlio Vargas (FGV/RJ), um da Universidade de Brasília (UnB), um do Centro Federal de Educação Tecnológica de Minas Gerais, um da Fundação Educacional Inaciana Padre Saboia de Medeiros (FEI/SP) e, por fim, um caracterizado com particular. Nesse contexto, pode-se afirmar que os estados que se destacam na elite em teoria institucional são: Paraná com cinco dos 15 (33\%); três de São Paulo (20\%); dois do Rio de Janeiro (13\%); dois de Minas Gerais (13\%); um do Rio Grande do Sul (7\%); um do Distrito Federal (7\%) e um sem informação (7\%).

O vínculo predominante entre os autores analisados ficou dividido entre dois com maior frequência e que correspondem juntos a $85,7 \%$, sendo 
aproximadamente $42,9 \%$ para cada, tanto celetista quanto servidor público, conforme é apresentado na Tabela 6.

\section{Tabela 6: Vínculo institucional informado pelo autor}

\begin{tabular}{ll|c|c|c}
\cline { 1 - 3 } \multicolumn{2}{c|}{ Tipo de Vínculo } & Qtde. & \%Freq. & \%Acum. \\
\cline { 1 - 3 } $\mathbf{1}$ & Celetista & 42 & $42,9 \%$ & $42,9 \%$ \\
$\mathbf{2}$ & Servidor Público & 42 & $42,9 \%$ & $85,7 \%$ \\
$\mathbf{3}$ & Bolsista & 3 & $3,1 \%$ & $88,8 \%$ \\
$\mathbf{4}$ & Colaborador & 3 & $3,1 \%$ & $91,8 \%$ \\
$\mathbf{5}$ & Autônomo & 2 & $2,0 \%$ & $93,9 \%$ \\
$\mathbf{6}$ & Sem Informação & 6 & $6,1 \%$ & $100 \%$ \\
\cline { 1 - 3 } \multicolumn{2}{c|}{ Total } & $\mathbf{9 8}$ & $\mathbf{1 0 0 \%}$ & \multicolumn{1}{c}{}
\end{tabular}

Fonte: Elaborada pelos autores, com base na coleta dos dados

Ao analisar-se a elite dos pesquisados, ou seja, os 15 que mais publicaram em teoria institucional no cenário brasileiro, oito possuem vínculo institucional na modalidade celetista, enquanto o restante possui vínculo de servidor público (6), predominando os dois vínculos já citados. Ainda, existe um com vínculo autônomo.

\subsubsection{Autoria e coautoria}

O número de autores por artigo apresentou resultados aparentemente normais, conforme a Tabela 7. Há uma concentração de coautoria apresentando o agrupamento de dois autores com 46,3\% do total (relação professor e aluno, por exemplo, aluno regular e professor orientador), seguido pelo agrupamento de um e três autores com 22,4\% cada. A quantidade maior de agrupamento dos artigos analisados foi com quatro autores com apenas $9 \%$ do total. Esse resultado reforça o argumento de que muitas parcerias de orientações continuam sendo importantes geradores de produção acadêmica (aluno regular e orientador). 
Tabela 7: Número de autores por artigo

\begin{tabular}{c|c|c|c|c|c}
\hline Descrição & $\mathbf{1}$ & $\mathbf{2}$ & $\mathbf{3}$ & $\mathbf{4}$ & Total \\
\hline Artigos & 15 & 31 & 15 & 6 & $\mathbf{6 7}$ \\
\% Freq. & $22,4 \%$ & $46,3 \%$ & $22,4 \%$ & $9,0 \%$ & $\mathbf{1 0 0 \%}$ \\
Autores & 15 & 77 & 122 & 146 & $\mathbf{1 4 6}$ \\
\% Freq. & $10,3 \%$ & $52,7 \%$ & $83,6 \%$ & $100,0 \%$ & $\mathbf{1 0 0 \%}$ \\
\hline
\end{tabular}

Fonte: Elaborada pelos autores, com base na coleta dos dados

Dentre os autores que se repetiram com as publicações, e que caracterizam a elite dos autores no tange à discussão da teoria institucional no cenário nacional, em ordem decrescente: Clóvis Luiz Machado-da-Silva (17 artigos); João Marcelo Crubellate (6 artigos); Luciano Rossoni (6 artigos); Fernando Dias Lopes (5 artigos); Edson Ronaldo Guarido Filho (4 artigos); Flávio Carvalho de Vasconcelos (4 artigos); Valéria Silva da Fonseca (4 artigos); Marcelo Milano Falcão Vieira (3 artigos); Afrânio Carvalho Aguiar (2 artigos); Carlos Alberto Pereira (2 artigos); Isabella Francisca Freitas Gouveia de Vasconcelos (2 artigos); Paulo Sérgio Grave (2 artigos); Reinaldo Guerreiro (2 artigos); Tomás de Aquino Guimarães (2 artigos) e Uajará Pessoa Araújo (2 artigos). Todos esses autores juntos contribuíram com $43,2 \%$ da produção total, enquanto os demais possuem apenas uma contribuição por autor, perfazendo um total de 56,8\%.

A lei de Lotka estabelece que a proporção daqueles que fazem uma única contribuição é de mais ou menos $60 \%$, número que se aproximou do resultado encontrado que foi $56,8 \%$, e, ainda, que uma larga proporção da literatura científica é produzida por um pequeno número de autores, e um grande número de pequenos produtores se iguala, em produção, ao reduzido número de grandes produtores, neste caso confirmado, pois são 43,2\% (grupo que publica muito) e 56,8\% (grupo que publica pouco). Assim, podese afirmar que, tendo em vista outros campos de pesquisa, a produção científica em teoria institucional é produtiva no Brasil.

\subsection{MAPEAMENTO GERAL DAS OBRAS CITADAS}

O segundo objetivo foi mapear as obras citadas pelos autores nas referências. Para tanto este item será subdividido em três subitens: primeiro 
será tratado o número de ocorrência das citações por obra; no segundo será apresentada a relevância das obras; e no terceiro realizar-se-á uma intersecção entre a frequência com que a obra é citada e a quantidade que aparece nos artigos.

\subsubsection{Citações das obras}

No que tange às citações (Tabela 8), podem-se destacar os seguintes pontos.

Tabela 8: Número de citações das obras utilizadas pelos autores dos artigos

\begin{tabular}{c|c|c|c}
\hline $\mathbf{N}^{\circ}$. de Citações das Obras & $\begin{array}{c}\mathbf{N}^{0} \text {. de Vezes que } \\
\text { Ocorrem Citações }\end{array}$ & $\begin{array}{c}\text { Total de } \\
\text { Citações }\end{array}$ & $\begin{array}{c}\mathbf{\%} \\
\text { Frequência }\end{array}$ \\
\hline $\mathbf{1}$ & 931 & 931 & $18,01 \%$ \\
$\mathbf{2}$ & 363 & 726 & $14,04 \%$ \\
$\mathbf{3}$ & 147 & 441 & $8,53 \%$ \\
$\mathbf{4}$ & 107 & 428 & $8,28 \%$ \\
$\mathbf{5}$ & 44 & 220 & $4,26 \%$ \\
$\mathbf{6}$ & 35 & 210 & $4,06 \%$ \\
$\mathbf{7}$ & 33 & 231 & $4,47 \%$ \\
$\mathbf{8}$ & 18 & 144 & $2,79 \%$ \\
$\mathbf{9}$ & 11 & 99 & $1,91 \%$ \\
$\mathbf{1 0}$ & 8 & 80 & $1,55 \%$ \\
$\mathbf{1 0}$ citações & --- & $\mathbf{3 . 5 1 0}$ & $\mathbf{6 7 , 8 9 \%}$ \\
Total até & -- & $\mathbf{1 . 6 6 0}$ & $\mathbf{3 2 , 1 1} \%$ \\
Total de 11 a 180 citações & --- & $\mathbf{5 . 1 7 0}$ & $\mathbf{1 0 0} \%$ \\
\hline
\end{tabular}

Fonte: Elaborada pelos autores, com base na coleta dos dados

a. As ocorrências perfazem um total de 5.170 citações, o que corresponde à média aritmética de aproximadamente 77 citações no decorrer do artigo.

b. As citações por obra variaram entre uma até 180 ocorrências por obra no somatório dos 67 artigos analisados.

c. As quantidades que mais se repetem são até quatro citações por texto, o que juntas somam $48,86 \%$ do total das vezes que ocorreram as citações. 
d. As sete obras mais citadas correspondem, respectivamente, na ordem decrescente: uma com 180 vezes; uma com 130; uma com 102; uma com 61; uma com 56; uma com 50; e uma com 44.

e. Assim, as citações ficaram distribuídas da seguinte forma: $67,89 \%$ até a ocorrência de dez vezes e de 11 a 180 ocorrências com $32,11 \%$.

f. Perfez-se um total de 1.763 referências distintas, as quais, acumuladas com as devidas repetições, somaram 2.669, o que corresponde à média aritmética de 39,84 referências utilizadas por artigo.

g. Por fim, vale ressaltar que, embora a qualidade dos periódicos seja indiscutível, alguns ainda apresentam "incoerências" de edição, pois foi encontrada uma média de duas referências listadas a cada artigo sem nenhuma utilização no decorrer do mesmo.

\subsubsection{Relevância das obras referenciadas}

No que tange a relevância das obras citadas (Tabela 9), pode-se tecer os comentários a seguir.

\section{Tabela 9: Relevância em quantidade das obras referenciadas}

\begin{tabular}{c|c|c|c|c|c|c|c|c|c|c|c|c|c|c|c|c|c|c}
\hline Descrição & $\mathbf{4 5}$ & $\mathbf{4 4}$ & $\mathbf{3 0}$ & $\mathbf{2 0}$ & $\mathbf{1 4}$ & $\mathbf{1 3}$ & $\mathbf{1 2}$ & $\mathbf{1 1}$ & $\mathbf{9}$ & $\mathbf{8}$ & $\mathbf{7}$ & $\mathbf{6}$ & $\mathbf{5}$ & $\mathbf{4}$ & $\mathbf{3}$ & $\mathbf{2}$ & $\mathbf{1}$ & Total \\
\hline Qtde. & 1 & 1 & 1 & 1 & 1 & 3 & 3 & 2 & 2 & 12 & 7 & 10 & 20 & 21 & 36 & 155 & 1.487 & $\mathbf{1 . 7 6 3}$ \\
\% Freq. do TT & $0,1 \%$ & $0,1 \%$ & $0,1 \%$ & $0,1 \%$ & $0,1 \%$ & $0,2 \%$ & $0,2 \%$ & $0,1 \%$ & $0,1 \%$ & $0,7 \%$ & $0,4 \%$ & $0,6 \%$ & $1,1 \%$ & $1,2 \%$ & $2,0 \%$ & $8,8 \%$ & $84,3 \%$ & $\mathbf{1 0 0} \%$ \\
\% Freq. & $67,2 \%$ & $65,7 \%$ & $44,8 \%$ & $29,9 \%$ & $20,9 \%$ & $19,4 \%$ & $17,9 \%$ & $16,4 \%$ & $13,4 \%$ & $11,9 \%$ & $10,4 \%$ & $9,0 \%$ & $7,5 \%$ & $6,0 \%$ & $4,5 \%$ & $3,0 \%$ & $1,5 \%$ & \\
\hline
\end{tabular}

Fonte: Elaborada pelos autores, com base na coleta dos dados

a. Foi encontrado um total de 1.763 obras diferentes.

b. A frequência mais encontrada, assim como nas citações, é de uma até três obras referenciadas no somatório dos 67 artigos, sendo 84,3\% para apenas a ocorrência de uma referência, 8,8\% para duas referências e $2 \%$ para três referências. 
c. As cinco obras mais relevantes foram referenciadas, respectivamente, em: uma em 45 artigos, ou seja, 67,2\% do total de 67; uma em 44 artigos (65,7\%); uma em 30 artigos (44,8\%); uma em 20 artigos (29,9\%); e uma em 14 artigos (20,9\%).

Como último ponto a discutir-se neste artigo, destacam-se, a seguir, a intersecção das citações e a relevância das obras encontradas na análise.

\subsubsection{Intersecção das citações e relevância}

Com base no exposto nos dois itens anteriores (4.2.1 Citações e 4.2.2 Relevância), este item tem como objetivo promover a intersecção entre a quantidade e a relevância das referências utilizadas pelos 67 artigos analisados, conforme apresentados no Quadro 4.

\begin{tabular}{|c|c|c|}
\hline \multicolumn{3}{|c|}{ Quantidade Total de Citações [Citações] } \\
\hline $1^{\circ}$ & $\begin{array}{l}\text { DiMaggio, P. J., \& Powell, W. T. (1983) [Versões } \\
\text { 1982, } 1991 \text { e } 2004 \text { em Inglês; } 2005 \text { em } \\
\text { Português] }\end{array}$ & 180 \\
\hline $2^{\circ}$ & $\begin{array}{l}\text { Meyer, J. W., \& Rowan, B. (1977) [Versões 1983, } \\
1991 \text { e } 1992 \text { em Inglês] }\end{array}$ & 130 \\
\hline & $\begin{array}{l}\text { Scott, R. W. (1995) [Versões } 2001 \text { e } 2008 \text { em } \\
\text { Inglês] }\end{array}$ & 102 \\
\hline $4^{\circ}$ & $\begin{array}{l}\text { Giddens, A. (1984) [Versões } 1989 \text { e } 2003 \text { em } \\
\text { Português] }\end{array}$ & 61 \\
\hline & $\begin{array}{l}\text { Tolbert, P. S., \& Zucker, L. G. (1996) [Versões } \\
1998 \text { e } 1999 \text { em Português; } 1997 \text { em Inglês] }\end{array}$ & 56 \\
\hline 60 & $\begin{array}{l}\text { Berger, P. L., \& Luckmann, T. (1967) [Versões } \\
\text { 1985, 1997, 1998, 2001, } 2002 \text { e } 2005 \text { em } \\
\text { Português; } 1966 \text { em Inglês] }\end{array}$ & 50 \\
\hline 70 & Oliver, C. (1991) & 44 \\
\hline & $\begin{array}{l}\text { Simon, H. (1947) [Versões 1965, 1970, 1979, } \\
1997 \text { em Português; 1945, } 1976 \text { em Inglês] }\end{array}$ & 38 \\
\hline
\end{tabular}

\begin{tabular}{|c|c|c|}
\hline \multicolumn{3}{|c|}{ Quantidade Total de Artigos [Relevância] } \\
\hline 10 & $\begin{array}{l}\text { Meyer, J. W., \& Rowan, B. (1977) [Versões 1983, } \\
1991 \text { e } 1992 \text { em Inglês] }\end{array}$ & 45 \\
\hline 20 & $\begin{array}{l}\text { DIMaggio, P. J., \& Powell, W. I. (1983) [Versoes } \\
1982,1991 \text { e } 2004 \text { em Inglês; } 2005 \text { em } \\
\text { Portwâns] }\end{array}$ & 44 \\
\hline 30 & $\begin{array}{l}\text { Scott, R. W. (1995) [Versões } 2001 \text { e } 2008 \text { em } \\
\text { Inglês] }\end{array}$ & 30 \\
\hline $4^{\circ}$ & $\begin{array}{l}\text { Berger, P. L., \& Luckmann, T. (1967) [Versões } \\
\text { 1985, 1997, } 1998,2001,2002 \text { e } 2005 \text { em } \\
\text { Português; } 1966 \text { em Inglês] }\end{array}$ & 20 \\
\hline 50 & $\begin{array}{l}\text { Machado-da-Silva, C. L., Fonseca, V. S., \& } \\
\text { Crubellate, J. M. (2005) [Versão RAC } 2005 \text { em } \\
\text { Português e na BAR 2005 em Inglês] }\end{array}$ & 14 \\
\hline & $\begin{array}{l}\text { Giddens, A. (1984) [Versões } 1989 \text { e } 2003 \text { em } \\
\text { Português] }\end{array}$ & 13 \\
\hline 70 & Oliver, C. (1991) & 13 \\
\hline & $\begin{array}{l}\text { Simon, H. (1947) [Versões 1965, 1970, 1979, } \\
1997 \text { em Português; 1945, } 1976 \text { em Inglês] }\end{array}$ & 13 \\
\hline
\end{tabular}

\section{Quadro 4: Intersecção das citações e relevância}

Fonte: Elaborado pelo autor, com base na coleta dos dados

Partindo-se da hipótese apresentada por Guedes e Borschiver (2005) de que, em determinada área científica, trabalhos citados mais frequentemente são considerados mais relevantes do que trabalhos menos citados, pode-se inferir que as quantidades de citações tabuladas demonstram os textos relevantes no campo da teoria institucional utilizada pelos autores que publicam artigos em periódicos nacionais, ou, no mínimo, que são textos que proporcionam uma fundamentação teórica relevante à discussão da teoria no campo das organizações. 
No que tange aos oito textos que mais receberam citações, confrontados com os oitos textos que foram mais referenciados nos artigos analisados, foi encontrada uma divergência em apensas uma obra, o que reafirma a utilização da citação para corroborar a relevância, conforme Quadro 4. Por fim, vale ressaltar que embora as posições sejam trocadas no que tange à citação e à relevância, os textos são os mesmos e convergem na posição 3, 7 e 8 . Ainda, os dois textos que não aparecem nas duas listas (citação e relevância) estão situados na mesma posição 5 (intermediário).

De forma geral, ao se analisarem as oito referências apresentadas na Tabela 10, ocorre que apenas uma obra é nacional: Machado-da-Silva, Fonseca e Crubellate (2005), situação que reforça a fala de Caldas (2007, p. 4), "por mitigar o problema mediante a sugestão de longas listas de leituras complementares, que compreendem em geral artigos e livros não disponíveis em português", ou seja, a literatura referência está na maioria das vezes em língua estrangeira. Embora esta obra esteja disponível na BAR em língua inglesa. Também importante resgatar a ressalva de Mota et al. (2010), que também acharam a replicação de textos consagrados internacionalmente, considerados clássicos na discussão de teoria institucional.

Por fim, pode-se evidenciar que o resultado encontrado neste artigo não difere daqueles de Machado-da-Silva, Cunha e Amboni (1990), Bertero e Keinert (1994) e Vergara e Carvalho Jr. (1995), que afirmam que a produção brasileira repete didaticamente o que é produzido no exterior. Os achados indicam que $100 \%$ dos autores mais utilizados e lidos, bem como os mais citados, são referências internacionais. E ainda, conforme Rossoni (2006, p. 23), "não é surpresa verificar uma grande permeabilidade da análise organizacional por outros temas, pois historicamente o campo da administração sempre foi marcado por contribuições de diversas áreas". De forma generalizada, muitos dos estudos chegaram a resultados semelhantes, apesar de avaliarem disciplinas distintas.

\section{CONSIDERAÇÕES FINAIS}

A partir deste estudo e de outros consultados para embasamento teórico, fica presente que a bibliometria vem se consolidando como método 
de estudo que se preocupa com leituras mais ricas da realidade, mais atentas às reivindicações contemporâneas do pensamento complexo, bem como proporciona acesso exclusivo àquilo que é mensurável como cientificamente relevante para determinado campo de conhecimento, que neste caso em específico teve como foco a teoria institucional em periódicos no cenário brasileiro.

Portanto, a partir deste estudo, pode-se concluir que o perfil dos autores que produzem conhecimento na área da teoria institucional no cenário nacional é composto com maior frequência pelas seguintes características: formação em administração ou engenharia; linha de pesquisa em estratégia organizacional ou estudos organizacionais / teoria organizacional; doutorado ou mestrado; gênero masculino; maior titulação obtida entre 2003 e 2010; vínculo institucional FGV/SP, PUC/PR, USP/SP ou UP/PR; e vínculo institucional celetista ou servidor público. Com base na hipótese de que poucos publicam muito e muitos publicam pouco (lei de Lotka), pode-se comprovar que $56,8 \%$ dos autores contribuíram apenas uma vez, enquanto 15 dos 69 correspondem a 43,2\% da produção total. A qual pode ser considerada como produtiva.

Ainda, segundo a Lei de Bradford, a Revista de Administração Contemporânea (RAC), classificada pela Capes como estrato $A 2$, compõe o core em teoria institucional no cenário nacional, pois representa $26,9 \%$ de toda a publicação em periódicos classificados pela Capes de A1 até B2. Esse periódico é seguido pela revista Brazilian Administration Review (BAR) com 19,4\%, classificada pela Capes como A2. Vale ressaltar que as duas revistas compõem o rol das revistas da Associação Nacional de Pós-Graduação e Pesquisa em Administração (ANPAD), considerada uma instituição responsável por fomentar as pesquisas e os programas de pós-graduação na área de administração em âmbito nacional, e que, de acordo com a nova avaliação da Capes, "ganharam" um estrato por serem periódicos que estimulam o crescimento da área e são de associações da área de administração.

A partir dos resultados, pode-se inferir que 0 incentivo ao desenvolvimento de pesquisas e programas de pós-graduação reflete diretamente na qualidade dos artigos publicados nos periódicos nacionais, tendo em vista a mão de obra, no caso o pesquisador, de cunho qualificado 
e crítico. Foi encontrada uma relação próxima de 1,19 título de mestrado ou doutorado para cada artigo publicado em periódicos nacionais classificados pela Capes como A1 até B2. Por fim, é possível concluir os ganhos da bibliometria no que tange ao mapeamento das obras mais relevantes na discussão dos artigos que utilizam teorias organizacionais, neste caso especificamente a teoria institucional, e ainda a possibilidade de definir o core da área e o colégio invisível apresentados no decorrer do texto.

Este estudo apresenta limitações. Limitações que são inerentes ao método bibliométrico, pois ao recorrer às análises estatísticas e contagens de citações e cocitações, e mesmo incorporando análises qualitativas e de conteúdo, pelo menos nos principais autores, pode ocorrer o risco de alguma autoria ficar de fora, até por conta do corte arbitrário que precisa ser feito. 0 estudo bibliométrico fica restrito as fontes disponíveis e neste caso foram os dados da base formada pelos periódicos listados no método. Para futuras pesquisas, tentar evidenciar outros estratos Capes, ampliando a base de análise, a qual minimizará as interferências, e se possível, juntá-los e/ou compará-los a esta pesquisa, contrapondo e/ou aproximando os resultados.

\section{REFERÊNCIAS}

Alvarado, U. R. (2002). A lei de Lotka na bibliometria brasileira. Ciência da Informação, 31(2), 14-20.

Araújo, C. A. (2006). Bibliometria: evolução histórica e questões atuais. Em Questão, 12(1), 11-32.

Baron, J. N., Dobbin, F. R., \& Jennings, P. D. (1986). War and Peace: the evolution of modern personnel administration in U.S. industry. American Journal of Sociology, 92(2), 350-383.

Berger, P. L., \& Luckmann, T. (2011). A construção social da realidade. Petrópolis: Vozes.

Bertero, C. O., \& Keinert, T. M. M. (1994). A evolução da análise organizacional no Brasil (1961-93). Revista de Administração de Empresas, 34(3), 81-90.

Caldas, M. P. (2007). Paradigmas em estudos organizacionais: uma introdução a série. In M. P. Caldas, \& C. O. Bertero (Eds.), Teoria das organizações, São Paulo: Atlas. 
Caldas, M. P., \& Bertero, C. O. (Eds.). (2007). Teoria das organizações. São Paulo: Atlas.

Cintra, R. F., Amâncio-Vieira, S. F., Suzuki, T., \& Costa, B. K. (2014). Stakeholder theory: análise nos periódicos brasileiros a partir da bibliometria. Revista Portuguesa e Brasileira de Gestão, 13(4), 43-55.

Cunha, J. A. C., Defina, D. A., \& Passador, J. L. (2014). Qualidade da produção científica no Brasil: um enfoque nos trabalhos premiados pelos congressos da ANPAD 2004-2012. Revista de Ciências da Administração, $16(38), 11-28$.

DiMaggio, P. J., \& Powell, W. (1983). The iron cage revisited: institutional isomorphism and collective rationality in organization fields. American Sociological Review, 48(2), 147-160.

Diniz, C. M., Junquilho, G. S., \& Carrieri, A. P. (2003). A ação dos stakeholders e as tendências às práticas isomórficas em organizações do setor minerosiderúrgico: "seja um bom vizinho e sente comigo na calçada". Anais do Encontro Anual da Associação Nacional de Pós-Graduação e Pesquisa em Administração - ENANPAD, 27, Atibaia, SP, Brasil.

Giddens, A. (1984). The constitution of society. Berkeley: University of California Press.

Guedes, V. L. S., \& Borschiver, S. (2005). Bibliometria: uma ferramenta estatística para a gestão da informação e do conhecimento, em sistemas de informação, de comunicação e de avaliação científica e tecnológica. Anais do Encontro Nacional de Ciência da Informação - CINFORM, 6, Salvador, BA, Brasil.

Machado-da-Silva, C. L., Cunha, V. C., \& Amboni, N. (1990). Organizações: o estado da arte da produção acadêmica no Brasil. Anais do Encontro Anual da Associação Nacional de Pós-Graduação e Pesquisa em Administração ENANPAD, 14, Belo Horizonte, MG, Brasil.

Machado-da-Silva, C. L., \& Gonçalves, S. A. (1999). Nota técnica: a teoria institucional. In S. Clegg et al. (Orgs.), Handbook de estudos organizacionais (vol. 1, pp. 220-226). São Paulo: Atlas.

Meyer, J. W., \& Rowan, B. (1977). Institutionalized organizations: formal structure as myth and ceremony. American Journal of Sociology, 83(2), 340-363.

Moran, M. R., Souza, F. F. A., Boaventura, J. M. G., Marinho, B. L., \& Fischmann, A. A. (2010). Alianças estratégicas: uma análise bibliométrica da produção científica entre 1989 e 2008. Revista de Ciências da Administração, 12(27), 42-62.

Moretti, S. L. A., \& Campanario, M. A. (2009). A produção intelectual brasileira em responsabilidade social empresarial sob a ótica da bibliometria. Revista de Administração Contemporânea, 13(edição especial), 68-86. 
Mota, F. P. B., Beck, C. G., Pereira, R. C. F., Lima, T. A. P., \& Vale, S. C. (2010). Tudo novo ou tudo 'de novo'? A utilização de teoria em estudos organizacionais brasileiros. Anais do Encontro de Estudos Organizacionais, 6, Florianópolis, SC, Brasil.

Oliveira, D. L., Ferreira, E. P. S., Carneiro, A. F., Costa, R. F., \& Porto, W. S. (2015). Sucesso de sistemas de informações na administração pública: proposta de um modelo exploratório. Future Studies Research Journal, $7(2), 63-95$.

Oliver, C. (1991). Strategic responses to institutional processes. Academy of Management Review, 16(1), 145-179.

Ribeiro, I., \& Serra, B. P. C. (2014). Tomada de decisão estratégica: uma análise das citações e cocitações até o ano de 2014. Anais do Encontro Anual da Associação Nacional de Pós-Graduação e Pesquisa em Administração - ENANPAD, 34, Rio de Janeiro, RJ, Brasil.

Rossetto, C. R., \& Cunha, C. J. C. A. (1999). A importância dos stakeholders no processo de adaptação estratégica: um estudo de caso na indústria da construção civil. Anais do Encontro Anual da Associação Nacional de PósGraduação e Pesquisa em Administração - ENANPAD, 23, Foz do Iguaçu, PR, Brasil.

Rossoni, L. (2006). A dinâmica de relações no campo da pesquisa em organizações e estratégia no Brasil: uma análise institucional. Dissertação de Mestrado, Universidade Federal do Paraná: Curitiba, PR, Brasil.

Scott, R. W. (1995). Institutions and organizations. Thousand Oaks: Sage.

Simon, H. (1947). Administrative behavior: a study of decision-making processes in administrative organization. New York: MacMillan.

Tolbert, S. P., \& Zucker, L. G. (1999). A institucionalização da teoria institucional. In S. Clegg, C. Hardy, \& W. Nord (Orgs.), Handbook de estudos organizacionais (vol. 3, pp. 196-219). São Paulo: Atlas.

Vergara, S. C., \& Carvalho Jr., D. S. (1995). Nacionalidade dos autores referenciados na literatura brasileira sobre organizações. Anais do Encontro Anual da Associação Nacional de Pós-Graduação e Pesquisa em Administração - ENANPAD, 19, João Pessoa, Paraíba.

Vieira, D. M., \& Gomes, R. C. (2014). Mudança institucional gradual e transformativa: a influência de coalizões de advocacia e grupos de interesses em políticas públicas. Organizações \& Sociedade, 21(71), 679694.

Yoshida, N. D. (2010). Análise bibliométrica: um estudo aplicado à previsão tecnológica. Future Studies Research Journal, 2(1), 52-84.

Zittei, M. V., \& Fernandes, F. C. (2015). E-serviços no governo eletrônico: análise bibliométrica de artigos internacionais. Future Studies Research Journal, 7(2), 43-62. 